\title{
Introducing Physical Boundaries in Virtual Environments
}

\author{
Pilar Herrero and Angélica de Antonio \\ Facultad de Informática. Universidad Politécnica de Madrid. \\ Campus de Montegancedo S/N. \\ 28.660 Boadilla del Monte. Madrid. Spain \\ \{pherrero, angelica\}@fi.upm.es
}

\begin{abstract}
In this paper, we present an application developed at the Universidad Politécnica de Madrid with the aim of introducing boundaries' deformations into virtual environments. These studies have been run from a geometrical point of view with the aim of analysing how boundaries can modify the shape of some of the key concepts introduced into one of the most successful awareness models in Computer Supported Collaborative Work (CSCW), known as the Spatial Model of Interaction (SMI).
\end{abstract}

\section{Introduction}

The Spatial Model of Interaction (SMI) [1], as its name suggests, uses the properties of space as the basis for mediating interaction. It allows objects in a virtual world to govern their interaction through some key concepts - such as medium, aura, awareness, focus, nimbus, adapters and boundaries.

In the SMI the focus was understood as the observing object's interest, the nimbus was introduced as the observed object's projection and boundaries were identified as those objects that divide the space into different areas or regions, controlling the interaction between all of them. An object's focus and nimbus, can be modified through boundaries.

The main concept involved in controlling interaction between objects is awareness. Awareness quantifies and qualifies the interaction between every pair of objects in a given medium, being manipulated via focus and nimbus.

In this paper we are going to analyse how boundaries can modify the shape of focus and nimbus and how these modifications depend not just on the kind of boundary but also on the boundary's shape. We are also going to introduce some of the algorithms developed at the Universidad Politécnica de Madrid with the aim of introducing these deformations inside several projects and applications.

\section{Physical Deformation}

Starting from previous implementations of these concepts [2,3] where focus has been implemented as a triangle and nimbus has been implemented as a circumference, we have made a couple of assumptions: the focus shape was a circular sector and the nimbus shape was a circumference. 


\subsection{Deforming the Object Focus}

The pseudo-code associated with this deformation was:

1. Detect the boundaries with which it is possible to interact.

2. Having determined these boundaries, for each one $\left(O_{i}\right)$, it is necessary to get:

a. The set of boundary vertices, which we have called the "Significant Points"

b. Having established the vertices, for each one $\left(V_{i j}\right)$ :

i. Define segments between the centre of the focus shape and the vertex $\left(V_{i j}\right)$.

ii. Having established the segments, calculate the straight line defined by each of these segments $\left(S_{i j}\right)$ and the other boundaries.

iii. Taking into account the set of straight lines obtained in the previous point, calculate which intersect with the focus shape.

iv. From the points of intersection calculated in the previous step, get the points that are the closest to the centre of the focus shape $(O C)$ and furthest away from $V_{i j}$. Each of these points will be called $P_{i j}$ and will be part of the deformed shape. If $V_{i j}$ is not coincident with $P_{i j}$, then $V_{i j}$ will also be part of the deformed shape. However, if there is any point of intersection closer to OC than $V_{i j}$, there will be no points of deformation related to this vertex, and the set of points obtained will be part of the set of "Final Points" used in the deformation.

3. From the previous step, we got all the points necessary to define the deformation of the focus shape. However, the points have to be ordered by an ordering algorithm to define the correct deformation shape.

4. It could be necessary to add some of the circular sector vertices to the list of "Final Points" (see section 2.1.2)

5. Divide the deformed circular sector shape into different simple figures like circular sectors and triangles to build the resulting deformed circular sector shape taking the previous points as starting points.

6. Finally, draw the deformed circular sector shape, tracing the outline of the figures that define the outline of the deformed circular sector shape (Figure 1).



Fig. 1. Deformed Focus

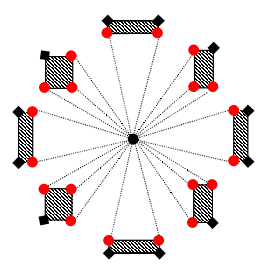

Fig. 2. Selected vertices with a circle and discarded vertices with a diamond

\subsubsection{Focus Significant Points}

Focus Significant Points are points that help to identify the shapes contributing to the deformed focus shape. Initially, significant points are determined by each of the boundaries that intersect with the deformed focus shape in the environment.

At first glance, one might think that these points are the vertices of these boundaries. However, this not the case, because depending on the boundary position 
related to the centre of the circular sector shape, we will have to discard the vertices that are hidden by the actual boundary (see the diamond vertices in Figure 2).

Having discarded these vertices, we also have to discard, from the remaining set of vertices, the vertices that are outside the circular sector shape. Instead, we are going to consider the points of intersection between the edges coming out of these vertices and the border of the circular sector shape (see the diamond vertices in Figure 3).

\subsubsection{Final Points}

The best way of understanding what points have to be considered as Final Points is by means of an illustration using a couple of examples.

If boundaries do not interfere with each other, then each of the boundaries is contributing independently to the set of final points (Figure 4). Moreover, it is necessary to add the three vertices of the circular sector.

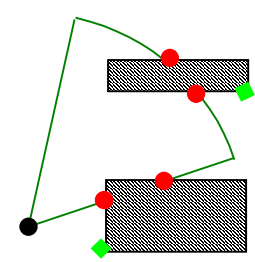

Fig. 3. Selected vertices with a circle and discarded vertices with a diamond

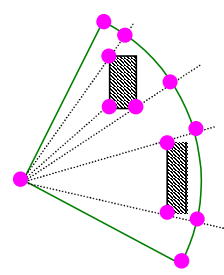

Fig. 4. Final Points

However, if a part of the object is in hiding, as is the case in the example shown in Figure 5, the final points provided by each of the boundaries have to be carefully analysed.

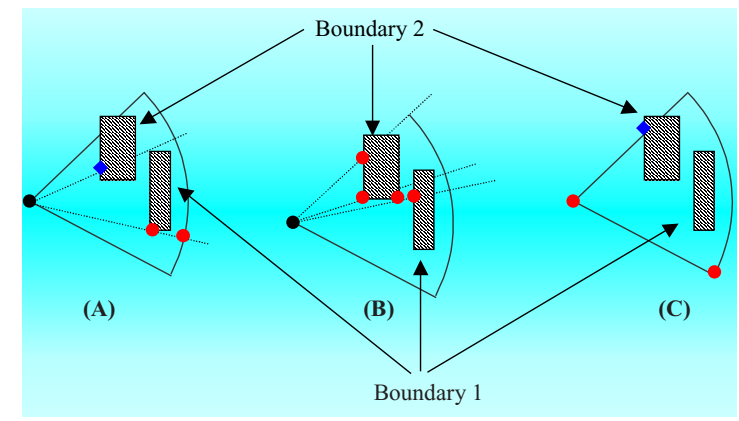

Fig. 5. Final Points with a circle

In Figure 5 (A), we can see that of all the final points that the boundary 1 should provide -4 points if no other boundary was interfering - just 2 of these points are contributing as final points, because of the presence of boundary 2 (the diamond point means that the other two points cannot be introduced). In Figure 5 (B), we can see the final points provided by boundary 2 , one of the final points provided by this boundary is on boundary 1's edge. In Figure 5 (C), we can see the final points provided by the vertex of the circular sector. From this figure, we can appreciate that the diamond 
point is obstructing the incorporation of the third vertex. The set of final points is the union of all the final points provided by each of these boundaries and the circular sector figure.

\subsubsection{Ordering Algorithm}

We have used a basic ordering criterion and some additional criteria for some specific situations that could occur while the application is running [4].

The basic ordering criterion is that given two points $P_{1}$ and $P_{2}, P_{1}$ is said to be greater than $P_{2}$ related to the origin of co-ordinates $O C$ and the origin angle $\alpha$ (Figure 6), and we write $P_{1}>_{O C \alpha} P_{2}$, if $\alpha_{1} \neq \alpha_{2}$ and $\alpha_{1}>\alpha_{2}$, where: $\alpha$ is the angle defined by the lower edge of the circular sector; $\alpha_{1}$ is the angle of the straight line defined by $O C-P_{1}$ dependent on $\alpha, \alpha_{2}$ is the angle of the straight line defined by $O C-P_{2}$ dependent on $\alpha$.

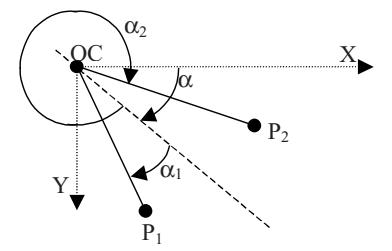

$\alpha_{1} \neq \alpha_{2}, \alpha_{2}>\alpha_{1} \rightarrow \mathrm{P}_{2}>_{\text {OC }} \mathrm{P}_{1}$

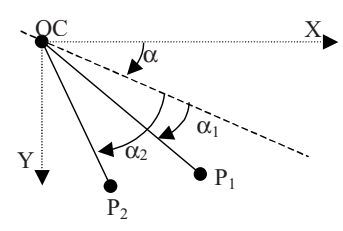

$\alpha_{1} \neq \alpha_{2}, \alpha_{2}>\alpha_{1} \rightarrow \mathrm{P}_{2}>_{\mathrm{OC}} \mathrm{P}_{1}$

Fig. 6. Ordering Criterion

\subsection{Deforming the Object Nimbus}

The sequence of steps in the pseudo-code is similar for deforming both focus and nimbus, the difference lying the internal procedure for completing each of the steps, that is, how the list of significant or final points is calculated. The pseudo-code for deforming the focus shape has another important difference: an additional step (number 4), which is not necessary for deforming the nimbus shape. The deformed circular shape is showed at the Figure 7.

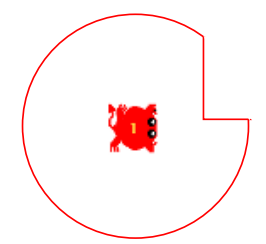

Fig. 7. Deformed nimbus

\subsubsection{Significant Points}

Significant points are the points that help to identify the shapes contributing to the deformed circular nimbus shape. Initially, significant points are determined by each of the boundaries that intersect with the deformed nimbus shape in the environment.

At first glance, one might think that these points are the vertex of this boundary. However, this not the case, because: 
Depending on the boundary position related to the centre of the circular shape, we will have to discard the vertices that are hidden by the actual boundary (see the diamond vertices in Figure 8).

Having discarded these vertices, we also have to discard, from the remaining set of vertices, the vertices that are outside the circular shape. Instead, we are going to consider the points of intersection between the edges coming out of these vertices and the border of the circular shape (see the diamond vertices in Figure 9).

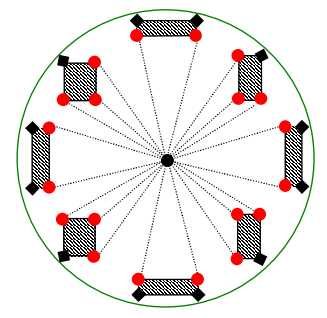

Fig. 8. Selected vertices with a circle and discarded vertices with a diamond

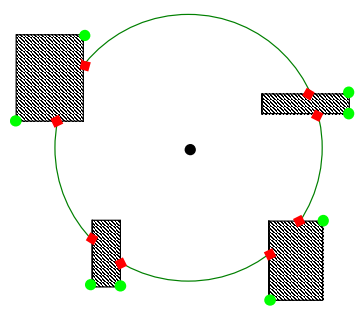

Fig. 9. Selected vertices with a diamond and discarded vertices with a circle

\subsubsection{Final Points}

The best way of understanding what points have to be considered as Final Points is by means of an illustration using a couple of examples.

If boundaries do not interfere with each other, then each of the boundaries is contributing independently to the set of final points (Figure 10).

However, if a part of the object is in hiding, as is the case in the example shown in Figure 11, the final points provided by each of the boundaries have to be carefully analysed. In Figure 11 (A), we can see that of all the final points that boundary 1 should provide -4 points if no other boundary was interfering - just 2 of these points are contributing as final points, because of the presence of boundary 2 (the diamond point means that the other two points cannot be introduced). In Figure 11 (B), we can see the final points provided by boundary 2 , one of the final points provided by this boundary is on boundary 1's edge. The set of final points is the union of all the final points provided by each of these boundaries.

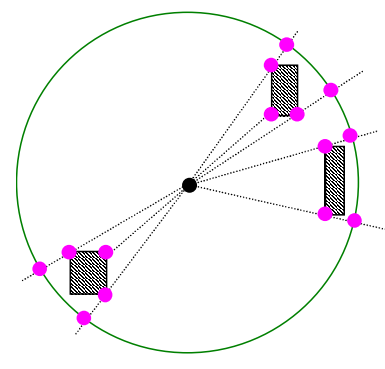

Fig. 10. Final Points



Fig. 11. Final Points with a circle 


\subsubsection{Ordering Algorithm}

We have used a basic ordering criterion and some additional criteria for some specific situations that could occur while the application is running [4].

The basic ordering criterion is that given two points $P_{1}$ and $P_{2}, P_{1}$ is said to be bigger than $P_{2}$ related to the origin of co-ordinates $O C$ (Figure 12), and we write $P_{1}$ $>_{O C} P_{2}$, if $\alpha_{1} \neq \alpha_{2}$ and $\alpha_{1}>\alpha_{2}$, where: $\alpha_{1}$ is the angle of the straight line defined by $O C-P_{1} ; \alpha_{2}$ is the angle of the straight line defined by $O C-P_{2}$.

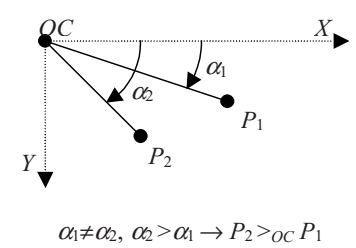

Fig. 12. Ordering criterion

\section{Conclusions}

We have developed an application to analyze how the presence of boundaries could modify some of the Key concepts of one of the most successful awareness models in Computer Supported Cooperative Work (CSCW), called the Spatial Model of Interaction (SMI) [1]. The SMI manages awareness in Collaborative Virtual Environments (CVEs) through these set of key concepts.

These studies have been run from a geometrical point of view with the aim of analysing how boundaries can modify the shape of two of key concepts introduced into the SMI - focus and nimbus - and how these modifications can have an effect on the awareness of interaction between each of them [4].

\section{References}

1. Benford, S., and Fahlén, L.E. A spatial model of interaction in large virtual environments, in Proc. Third European Conference on Computer Supported Cooperative Work (ECSCW'93), Milano, Italy. Kluwer Academic Publishers, pp. 109-124.

2. Greenhalgh, C. Dynamic, embodied multicast groups in MASSIVE-2, Technical Report NOTTCS-TR-96-8, Department of Computer Science, University of Nottingham, UK, 1996.

3. Greenhalgh, C., Large Scale Collaborative Virtual Environments, Doctoral Thesis. University of Nottingham. October 1997.

4. Herrero P. A Human-Like Perceptual Model for Intelligent Virtual Agents PhD Thesis. Universidad Politécnica de Madrid, June 2003. 